\title{
Aspirin induced long non coding RNA suppresses colon cancer growth
}

\author{
Mingwei Chen ${ }^{1 \#}$, Lili Wu ${ }^{2 \#}$, Huajie Zhan ${ }^{3 \#}$, Tonghua Liu' ${ }^{2}$ Yan $\mathrm{He}^{3}$ \\ ${ }^{1}$ Department of Human Anatomy, Harbin Medical University, Harbin, China; ${ }^{2}$ Tibet Medical College, Beijing University of Chinese Medicine, \\ Tibet, China; ${ }^{3}$ Department of Department of Pathology, Harbin Medical University, Harbin, China \\ Contributions: (I) Conception and design: Y He; (II) Administrative support: L Wu; (III) Provision of study materials or patients: M Chen; (IV) \\ Collection and assembly of data: H Zhan; (V) Data analysis and interpretation: T Liu; (VI) Manuscript writing: All authors; (VII) Final approval of \\ manuscript: All authors. \\ \#These authors contributed equally to this work. \\ Correspondence to: Yan He. Department of Department of Pathology, Harbin Medical University, Harbin 150081, China. Email: he_yan419@163. \\ com; Tonghua Liu. Tibet Medical College, Key Laboratory of Ministry of Education, Beijing University of Chinese Medicine, Tibet 850010, China. \\ Email: thliu@vip.163.com.
}

\begin{abstract}
Background: To study the effect of long non-coding RNAs (lncRNA) on aspirin-treated colon cancer cells. Methods: To study the changes of lncRNA in aspirin-treated colon cells by microarray analysis, real-time quantitative PCR (qPCR) was used to verify the expression of selected lncRNA and mRNA. The effects of aspirin on the proliferation and metastasis of the siRNA transfected cells and control colon cancer cells were detected by Cell Counting Kit-8 (CCK-8) and Transwell experiments. Bioinformatics tools were employed to analyze the potential function of lncRNA.
\end{abstract}

Results: Compared with the control group, aspirin inhibited the proliferation and metastasis of colon cancer cells. Microarray analysis showed that a total of 10,568 lncRNAs and 22,126 mRNAs were noticeably expressed in the aspirin-treated group $(\geq 1.5$-fold, $\mathrm{P}<0.05)$. The qPCR results showed that lncRNA and mRNA expressions were consistent with microarray analysis. The analysis of the co-expression network profiles of 58 lncRNA and $101 \mathrm{mRNA}$ differential genes showed a total of 158 nodes and 791 connections. Analysis of Gene Ontology (GO) and Kyoto Encyclopedia of Genes and Genomes (KEGG) pathways revealed that a variety of lncRNAs (NEAT1, LOC152578) were involved in the inhibition mechanism of aspirin in colon cancer.

Conclusions: lncRNA NEAT1 and LOC152578 are involved in the inhibition of tumor cell growth and metastasis by aspirin. The results of these analyses will help us further understand the mechanism of action of aspirin and the roles of lncRNAs in the prevention and treatment of colon cancer.

Keywords: Colon cancer (CRC); aspirin; lncRNA

Submitted Jun 02, 2020. Accepted for publication Apr 02, 2021.

doi: $10.21037 /$ tcr-20-2248

View this article at: http://dx.doi.org/10.21037/tcr-20-2248

\section{Introduction}

Colorectal cancer (CRC) is the third most common cancer in the world, and the most common type is colon adenocarcinoma (CA) (1). It was estimated that approximately 1 in 18 people would eventually develop CRC in their lifetime, and $40 \%$ of whom would die within five years of diagnosis, mainly due to late diagnosis $(2,3)$. The development of the CRC is a slow process, and it takes several years to invade and metastasize from atypical hyperplasia, polyps, adenomas, and CA $(4,5)$. Chronic inflammation is one of the causes of CRC (6). Aspirin is a classic non-steroidal anti-inflammatory drug (NSAID) 
that has been used in a wide range of conditions, including fever, pain. and inflammatory diseases (7). Recent studies show that long-term and low-dose aspirin can reduce the incidence of cancer, delay the malignant transformation process, reduce the risk of tumor metastasis, and cancer mortality (8-14). Although the beneficial aspects of aspirin for cancer patients have been widely recognized, the mechanism of its effect remains unclear. Previous studies have confirmed that aspirin's anticancer effect is attributed to the inhibition of COX-2, which is upregulated in a variety of cancer cells $(15,16)$. It is worth noting that there is increasing evidence that aspirin may also exert anticancer effects in a COX-independent manner. Long non-coding RNA (lncRNA) is an RNA molecule that is about 200 nucleotides long and does not translate protein functions. IncRNAs are similar to mRNAs in that they are usually transcribed by RNA polymerase II, 5' endcapped, 3' polyadenylation, and splicing multiple exons by classical genome splicing motifs (17-19). lncRNA has been shown to participate in a variety of biological processes (BP) such as transcription, translation, splicing, intracellular and extracellular transport, and has been implicated in a variety of diseases. IncRNA can interact with proteins, DNA, and RNA, to participate in all levels of gene regulation, including epigenetics, transcription, and post-transcriptional regulation (17,20-22). Many lncRNAs have been shown to play critical roles in the development of various diseases or identified as important biomarkers in diagnosis and treatment $(23,24)$. At the same time, genomewide association analysis of various tumor samples has revealed many $\operatorname{lncRNAs}$ associated with multiple types of cancers, including CRC (25). Previous studies have shown that multiple lncRNAs are involved in the occurrence and development of colon cancer. Some lncRNAs, such as CCAL, CASC11, CCAT2, H19, and HOTAIR (26-28), were overexpressed in CRC tissues and cells. Patients with high expression of these lncRNAs have a higher incidence of distant metastases and a lower survival rate. Although the molecular mechanism of aspirin's anti-cancer effect has been studied extensively, the involvement of many cellular components (CC), such as long non-coding RNAs, is not fully understood. This study attempts to answer these questions by looking into the expression differences of lncRNA and mRNA in aspirin-treated colon cancer cells. We present the following article in accordance with the MDAR checklist (available at http://dx.doi.org/10.21037/ tcr-20-2248).

\section{Methods}

\section{Cell culture and aspirin treatment}

Human CRC cell lines HCT116, SW620, and DLD1 were purchased from the American Type Culture Collection (ATCC, China), and cultured in DMEM (Hyclone, Logan, Utah, USA) (Carlsbad, California, USA) containing $100 \mathrm{IU} / \mathrm{mL}$ penicillin and $100 \mu \mathrm{g} / \mathrm{mL}$ streptomycin. For all studies, colon cells were incubated at $37{ }^{\circ} \mathrm{C}, 5 \% \mathrm{CO}_{2}$, and 90-95\% of relative humidity. Aspirin was purchased from Sigma (Sigma-Aldrich, St. Louis, Missouri, USA), and a $1 \mathrm{M}$ stock solution (dissolved in ethanol) was prepared.

\section{Cell migration assay}

For the detection of cell migration capacity, a 24-well chamber with $8 \mu \mathrm{m}$ wells was used. First, $1 \times 10^{5}$ cells were seeded in DMEM medium in the upper chamber (excluding serum). $700 \mu \mathrm{L}$ medium containing 10\% Fetal Bovine Serum (FBS) was then added to the lower chamber. After 24 hours of incubation at $37{ }^{\circ} \mathrm{C}$, the cells in the upper chamber were carefully removed with a cotton swab. Cells that passed through the membrane were fixed with methanol and then stained with $0.5 \%$ crystal violet. For quantification, cell counts were performed under a microscope at $\times 100$ magnification from eight randomly selected fields. Transiently transfect hNEAT 1 and LOC152578 siRNA into colon cancer cell line SW620 and HCT116. After 48 hours, the effect of siRNA on the migration ability of colon cancer cells was verified.

\section{Microarray analysis}

Gene microarray analysis was performed on colon cells treated with aspirin to detect differentially expressed lncRNA and mRNA. Approximately 10,568 lncRNAs and 22,126 coding transcripts were detected by the Arraystar Human lncRNA Microarray V3. The cell preparation and microarray hybridization were performed using an Agilent Gene Expression Hybridization Kit (Agilent Technologies, Santa Clara, California, USA). The array was then scanned using an Agilent microarray scanner and analyzed using The GeneSpring v.13.1 software (Agilent Technologies).

\section{Real-time qRT-PCR assay}

Total RNA was extracted from samples using TRIzol 
Table 1 Primers and siRNA sequences

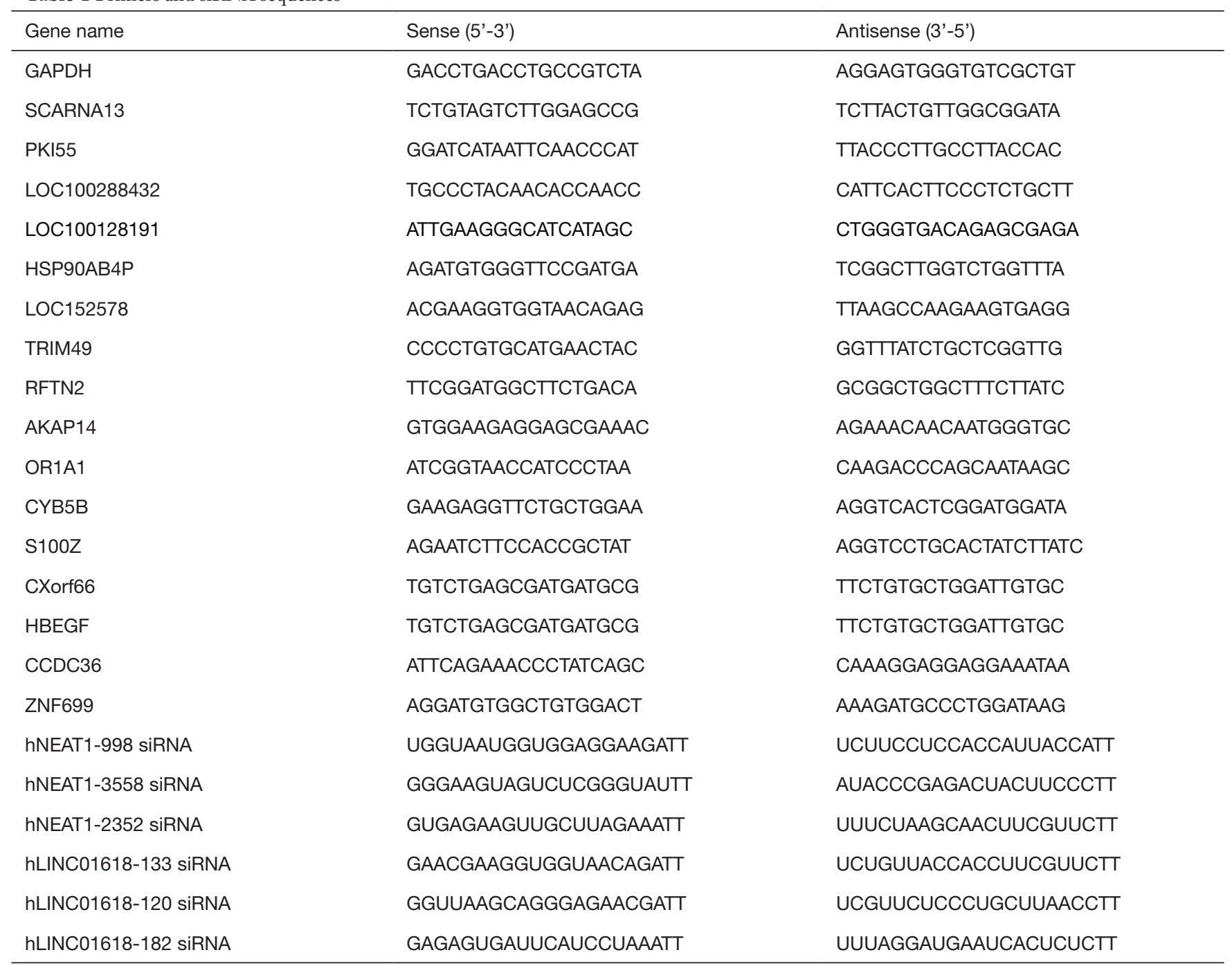

(Invitrogen, Carlsbad, California, USA). Detection of relative gene expression by SYBR Green PCR Mix (BIORESEARCHER, Beijing, China) and LightCycler 96 Real-Time System.

The qRT-PCR was performed on (Roche LC96). The thermal cycling program was $95^{\circ} \mathrm{C}$ for $5 \mathrm{~min}$, followed by 40 cycles of denaturation at $95^{\circ} \mathrm{C}$ for $10 \mathrm{~s}$, and amplification at $60{ }^{\circ} \mathrm{C}$ for $1 \mathrm{~min}$. Melting curve analysis was performed by progressive heating from 65 to $95^{\circ} \mathrm{C}$. The relative expression level of lncRNA or mRNA was calculated using the $2^{-\Delta \Delta C t}$ method and standardized for GAPDH. The primer sequences are summarized in Table 1. Data represent the average of three independent experiments.

\section{Identification of differentially expressed genes (DEGs)}

Probes with more than one gene and empty probes were discarded according to the annotation platform of each expression profile. We then extracted the expression profiles of lncRNA and mRNA, respectively. Three different aspirin-treated colon cells were compared with control. Finally, $\mathrm{P}$ value and $\log 2(\mathrm{FC})$ value of the two expression profiles were calculated, and a paired $t$-test was used to recognize DEGs. Statistically significant DEGs were 
defined as $|\log 2(\mathrm{FC})|<\log 2(1.5)$ and $\mathrm{P}<0.05$.

\section{siRNA transfect and cell counting kit-8 (CCK8)}

The hNEAT1 and LOC152578 siRNA [Sangon Biotech, (Shanghai) Co., Ltd.] transfected cells and control cells were seeded in 96 well plates and cultured for 0, 24, 48 and $72 \mathrm{~h}$, respectively. In order to transiently transfect siRNA into colon cancer cell line SW620, we first identified the putative promoter region of the target gene, then selected the reporter gene and the corresponding reporter gene analysis method, and finally inserted the promoter into the upstream of the reporter gene of the appropriate vector. After 48 hours, the effect of siRNA on the migration ability of colon cancer cells was verified. $10 \mu \mathrm{L}$ CCK8 was added to each well and cultured at $37{ }^{\circ} \mathrm{C}$ and $5 \% \mathrm{CO}_{2}$. The optical density (OD) value was measured at $450 \mathrm{~nm}$. The proliferation activity of SW620 cells was detected at 0, 24, 48 and $72 \mathrm{~h}$ after siRNA overexpression (Table 1).

\section{Construction of a co-expression network with $G O$ and KEGG analysis}

In order to identify the interaction between differentially expressed lncRNA and mRNA, a co-expression network was constructed using Cytoscape software, with validated lncRNA and related mRNA based on correlation analysis. Pearson's correlation coefficient was no less than 0.9. Pathway analysis was used to study important signaling pathways for DEGs. GO analysis was used to investigate the biological effects of aberrantly expressed mRNAs in three aspects: BP, molecular functions (MF), and CC.

\section{Statistical analyses}

All data are represented as mean \pm SD. Graphpad Prism 5.0 (San Diego, California, USA) was used for statistical analysis. The $t$-test was used to analyze the differences between the control and aspirin-treated data in this study. Pearson's correlation analysis is used to detect the relationship between lncRNA and mRNA. $\mathrm{P}<0.01$ is used as a threshold to define a GO term/pathway that is significantly enriched.

\section{Results}

\section{Aspirin inhibits colon cancer cell proliferation and metastasis}

The concentrations of aspirin used in this study ranged from 1 to $15 \mathrm{mM}$, based on past studies (29). The inhibitory effect of aspirin was determined by cell growth. By treating HCT116, DLD1, and SW620 cells with different concentrations of aspirin, aspirin inhibited colon cancer cell proliferation in a dose-dependent manner. When $50 \%$ of the cells were inhibited, the concentrations of aspirin were approximately 5,5 , and $3 \mathrm{mM}$, respectively (Figure 1A). The migration ability of the colon cells was also inhibited by the same concentrations (Figure 1B,C). These concentrations of aspirin were used in subsequent experiments for the three cell lines. Furthermore, when NEAT1 was knocked down, the cell proliferation rate decreased, indicating that NEAT1 could promote the proliferation of SW620 cell line. Aspirin has been reported to inhibit the growth of colon cancer cell line NEAT1. So, our experimental results are consistent with previous reports.

\section{lncRNA and mRNA expression analysis in aspirin-treated colon cells}

To compare the expression differences between lncRNA and mRNA in aspirin-treated colon cells, microarray analysis was employed to assess their expression levels. The expression profile heatmap was then generated with $\mathrm{R}$ language (Figure $2 A, B$ ). Next, we calculated the differentially expressed lncRNA and mRNA in three types of colon cells treated with aspirin, using $|\log 2(\mathrm{FC})|<\log 2(1.5)$ and $\mathrm{P}<0.05$ as the significance threshold. As shown by the volcanic maps, 58 significantly dysregulated lncRNAs were identified in the aspirin-treated group, with 28 being upregulated (red dots, Figure $3 A$ ) and 30 downregulated (green dots, Figure $3 A$ ). In comparison, 101 mRNAs were found to be significantly dysregulated, with 56 being upregulated (red dots, Figure 3B) and 45 downregulated (green dots, Figure $3 B$ ). Forty lncRNAs and 40 mRNAs with the most significant differential expressions are summarized in Table 2 and Table 3, respectively.

\section{Validation of the microarray data using $q R T-P C R$}

Ten mRNAs and 6 lncRNAs were randomly selected for qRT-PCR analysis to verify the results of microarray analysis. Consistent with the microarray analysis results, 5 of the selected mRNAs (TRIM49, RFTN2, AKAP14, OR1A1 and CYB5B) of the aspirin-treated group were shown to be upregulated, while the other five mRNAs (S100Z, CXor66, HBEGF, CCDC36 and ZNF699) downregulated. Furthermore, qRT-PCR analysis showed that lncRNA 
A

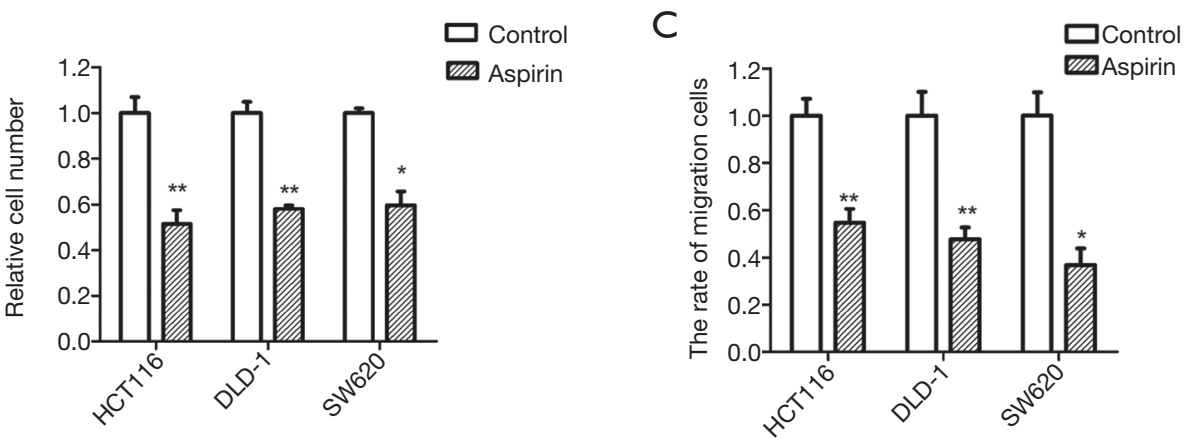

B

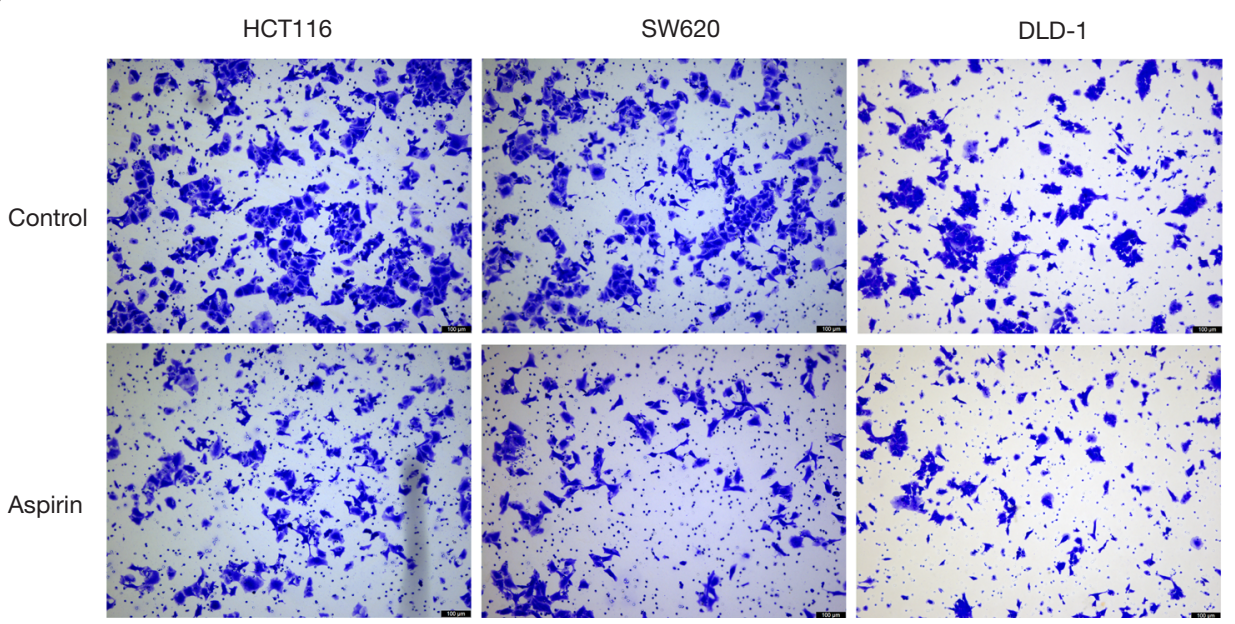

Figure 1 Aspirin inhibits colon cancer cell proliferation and metastasis. (A) The IC50 of aspirin on HCT116, SW620, and DLD1 cell proliferation were 5,5 , and $3 \mathrm{mM}$, respectively. (B) Three cell lines were treated with aspirin at the same concentrations for migration assays. Representative images show the inhibition of migration by aspirin in three colon cell lines. (C) Statistical results of migration assays. ${ }^{*} \mathrm{P}<0.05,{ }^{*} \mathrm{P}<0.01$ (Dyeing method: $0.5 \%$ Crystal violet; dyeing scale: $\times 100$ ).

SCARNA13, PKI55, LOC100288432 and LOC100128191 were upregulated, while lncRNAs HSP90AB4P and LOC152578 downregulated (Figure 4), confirming the validity of the microarray results. Both analyses provided compelling evidence that these lncRNAs and mRNAs may be involved in the pathogenesis of aspirin-treated colon cancer.

\section{GO term enrichment analysis}

GO term enrichment analysis results were presented with a bubble chart (Figure 5). DEGs were significantly enriched in GO cell components (CC), such as mitochondrial part, organelle envelope, mitochondrial membrane, and mitochondrial envelope. For MF, the DEGs were enriched in RNA binding and phosphatase inhibitor activity. In addition, BP analysis also displayed that the DEGs enriched in detection of stimulus involved in sensory perception (Figure 5).

\section{Co-expression analysis and construction of $\operatorname{lncRNA-mRNA}$ network}

To analyze the co-expression profile of lncRNA and mRNA, colon cells treated with aspirin were determined for each possible lncRNA-mRNA pair in the expression data. First, the lncRNAs or mRNAs which were differentially expressed were extracted. By using Pearson correlation coefficient (PCC), we find the correlation expression of lncRNA and mRNA PCC $>0.9$ and $\mathrm{P}<0.01$ was set as the threshold. A total of 169 DEGs (58 lncRNA and $101 \mathrm{mRNA}$ ) was filtered into the DEGs PPI network complex, containing 158 nodes and 791 edges. IncRNA-mRNA co-expression network was visualized by Cytoscape software (Figure 6). 

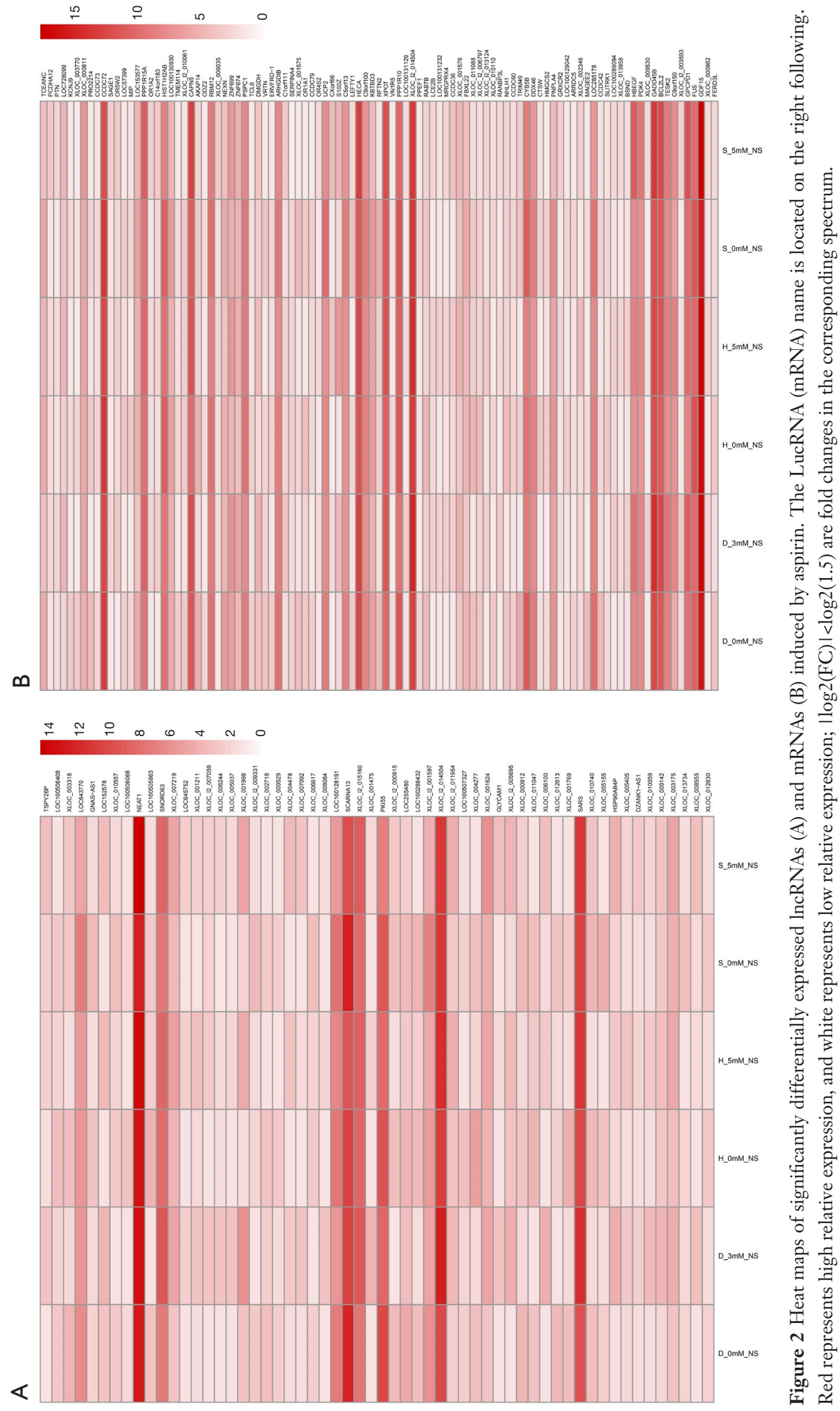

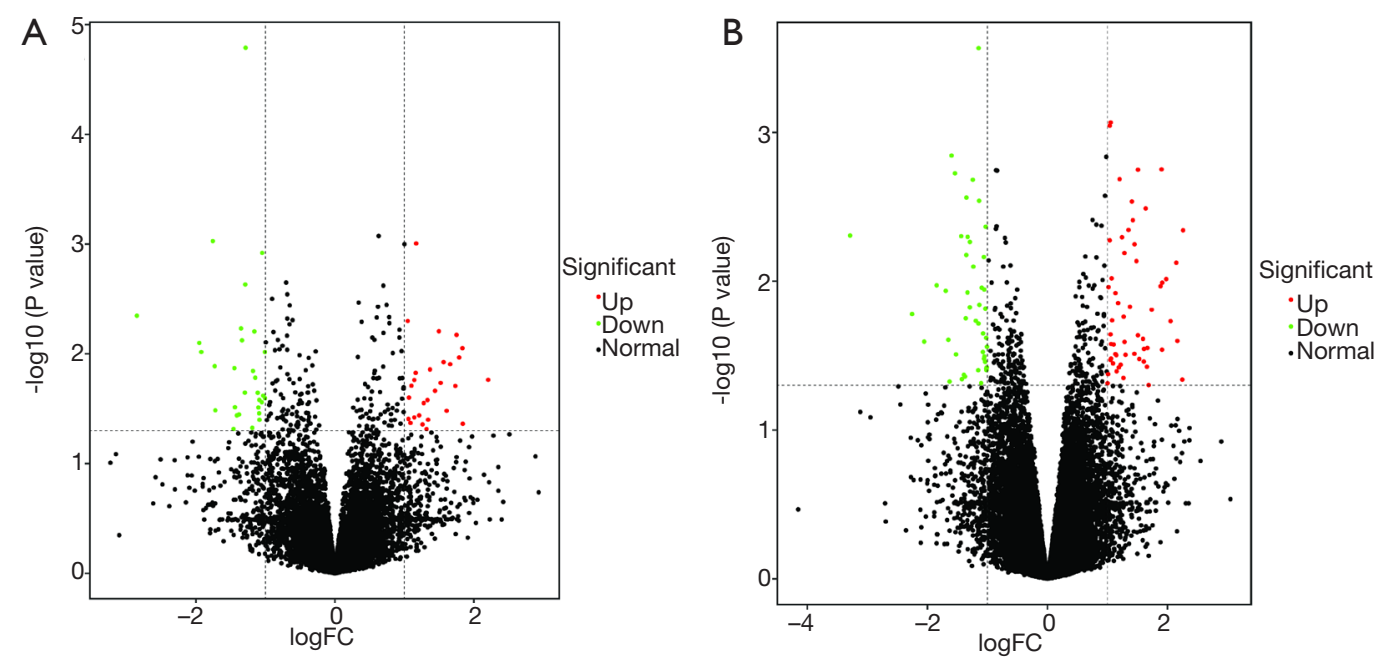

Figure 3 Volcano plots of differentially expressed genes. Scatter plot of lncRNA (A) and mRNA (B) expression variation between the aspirin-treated colon cells and control group. Red, upregulated $(\mathrm{FC}>1.5, \mathrm{P}<0.05)$; green, downregulated $(\mathrm{FC}<1.5, \mathrm{P}<0.05)$; black, nondifferentially expressed.

\section{Effects of NEAT1 and LOC152578 siRNA on proliferation and migration of colon cancer cell lines}

In order to study the effects of NEAT1 and LOC152578 siRNA on the proliferation and migration of colon cancer cell lines, we carried out CCK8 and Transwell experiments. First, we verified the success of siRNA knockdown by PCR (Figure 7A). Next, we tested the effects of NEAT1 and LOC152578 knockdown on the proliferation of colon cancer cell lines. The results showed that NEAT1 could promote the proliferation of SW620 cells, while LOC gene knockout did not inhibit the proliferation of SW620 cells (Figure $7 B$ ). In addition, the metastatic ability of SW620 and HCT116 cells was inhibited by knockdown of NEAT1 and LOC (Figure 7C). Aspirin has been reported to inhibit the growth of colon cancer cell line NEAT1. Therefore, our experimental results are consistent with previous reports (Figure 7D).

\section{Discussion}

There has been a growing number of studies in recent years indicating that not only can aspirin prevent several types of cancer, but also reduce the incidence of cancer and significantly inhibit the growth and promote apoptosis of cancer cells. Studies have shown that taking aspirin regularly can significantly reduce the incidence of CRC, factors affecting its efficacy including the time and dose of aspirin as well as the genetic background of the patient
(30,31). The antitumor effect of aspirin is mainly due to its inhibition on several major signaling pathways that promote cancer progression, such as COX/PGE2, PI3K/ AKT/mTOR, NF-кB, WNT/ $\beta$-catenin, and MAPK signaling pathways $(32,33)$. IncRNA plays a key role in gene expression regulation at both the transcriptional and the post-transcriptional levels, leading to a wide range of biological processes, such as tumorigenesis, growth, and metastasis in different human diseases, including cancer (34-36).

In this study, we investigated the changes of lncRNA and mRNA expression profiles in response to aspirin treatment in different CRC cells (DLD1, SW620 and HCT116) through microarray analysis. By using bioinformatics methods to analyze the common differential genes, 58 lncRNAs and 101 mRNAs were found to be significantly dysregulated in aspirin-treated colon cells. Further analysis revealed that 28 of the 58 dysregulated lncRNAs were upregulated and 30 downregulated, in response to the aspirin treatment in CRC cells. In the microarray results, the expression of lncRNA NEAT1, LOC152578, GLYCAM1, and SARS was markedly downregulated, compared to their expressions in human colon cancer without aspirin treatment $(37,38)$.

Traditionally a transcriptional regulator, NEAT1 (nuclear enriched abundant transcript 1) and the ribonucleoprotein complexes around NEAT 1 form the paraspeckles, a type of subnuclear body that is found adjacent to nuclear 
Table 2 Top 20 up and down expressed mRNAs in microarray analysis

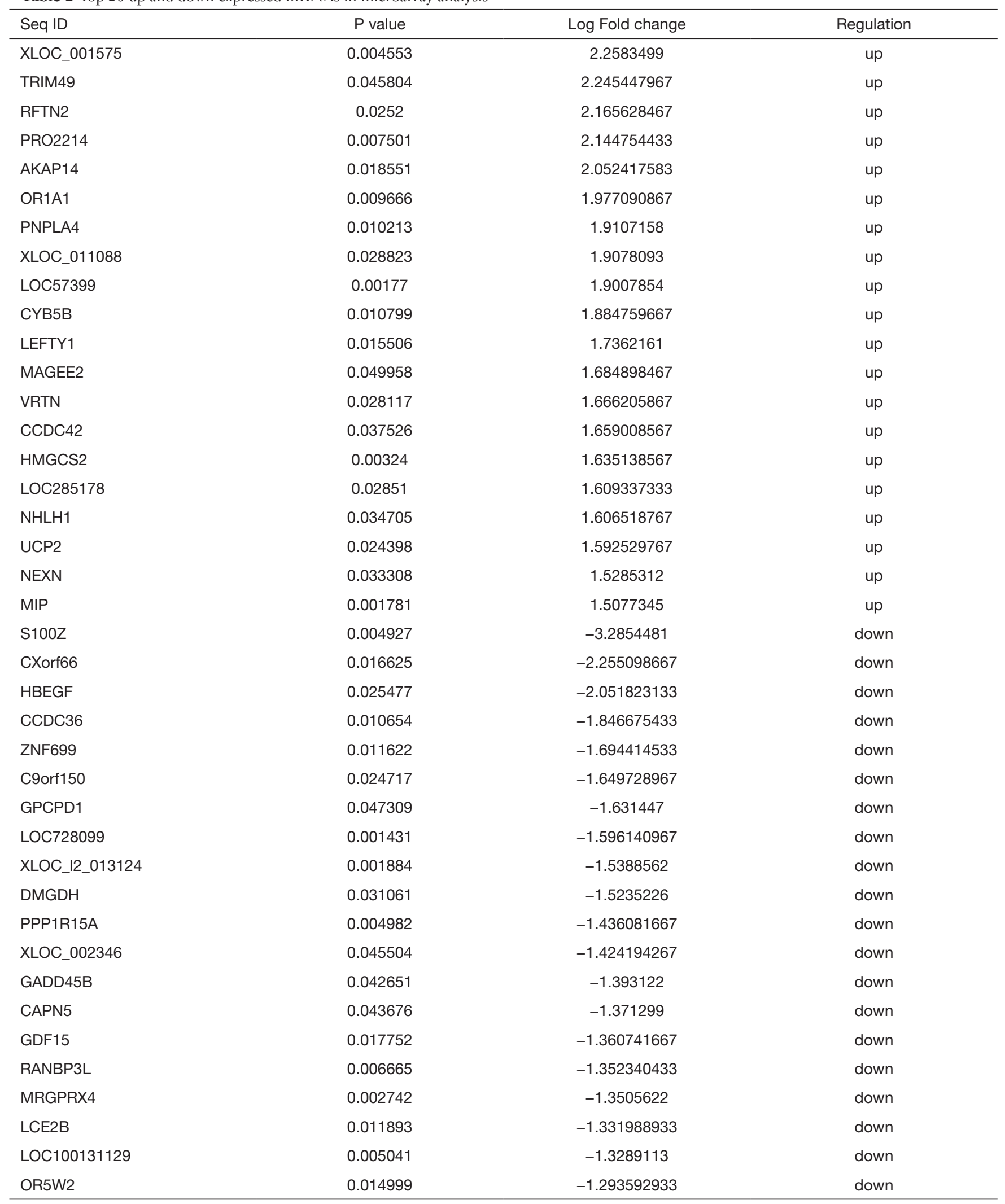


Table 3 Top 20 up and down expressed lncRNAs in microarray analysis

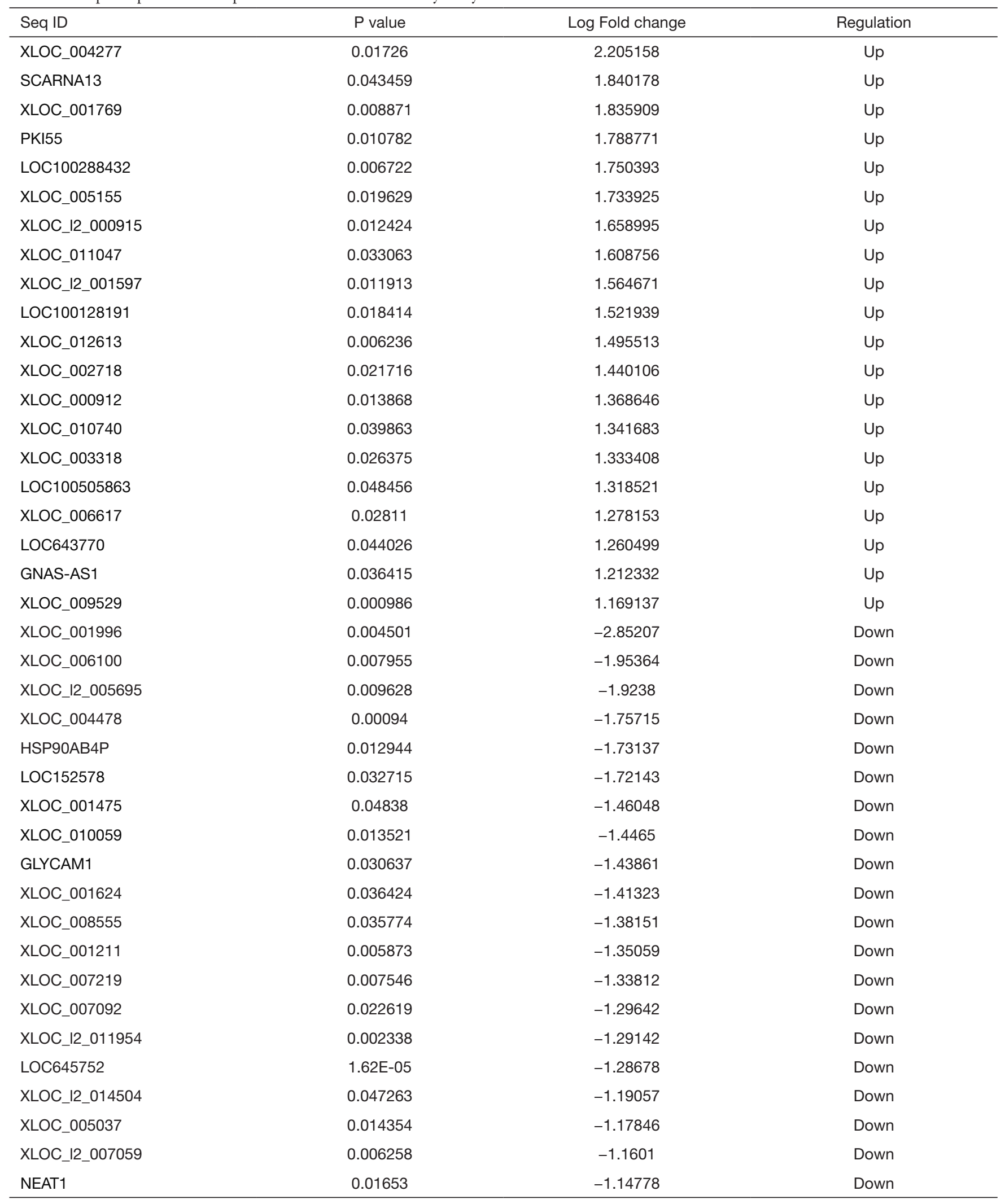




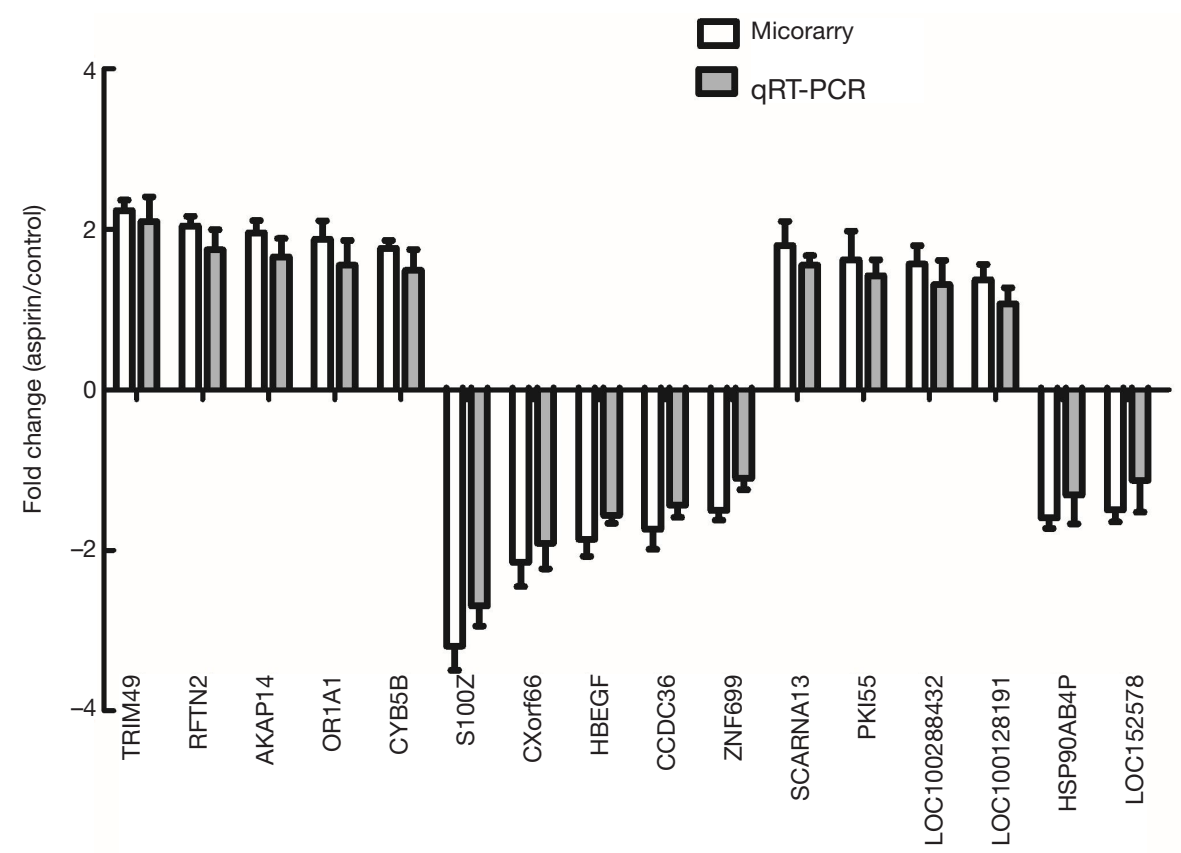

Figure 4 lncRNA and mRNA expression verified by qRT-PCR. Consistent with the microarray assay data, qRT-PCR results show that the expression levels of four lncRNAs (SCARNA13, PKI55, LOC100288432 and LOC100128191) and five mRNAs (TRIM49, RFTN2, AKAP14, OR1A1 and CYB5B) were upregulated in the aspirin-treated group when compared with the control. Two other lncRNAs (HSP90AB4P and LOC152578) and five other mRNAs (S100Z, CXor66, HBEGF, CCDC36 and ZNF699) were downregulated.

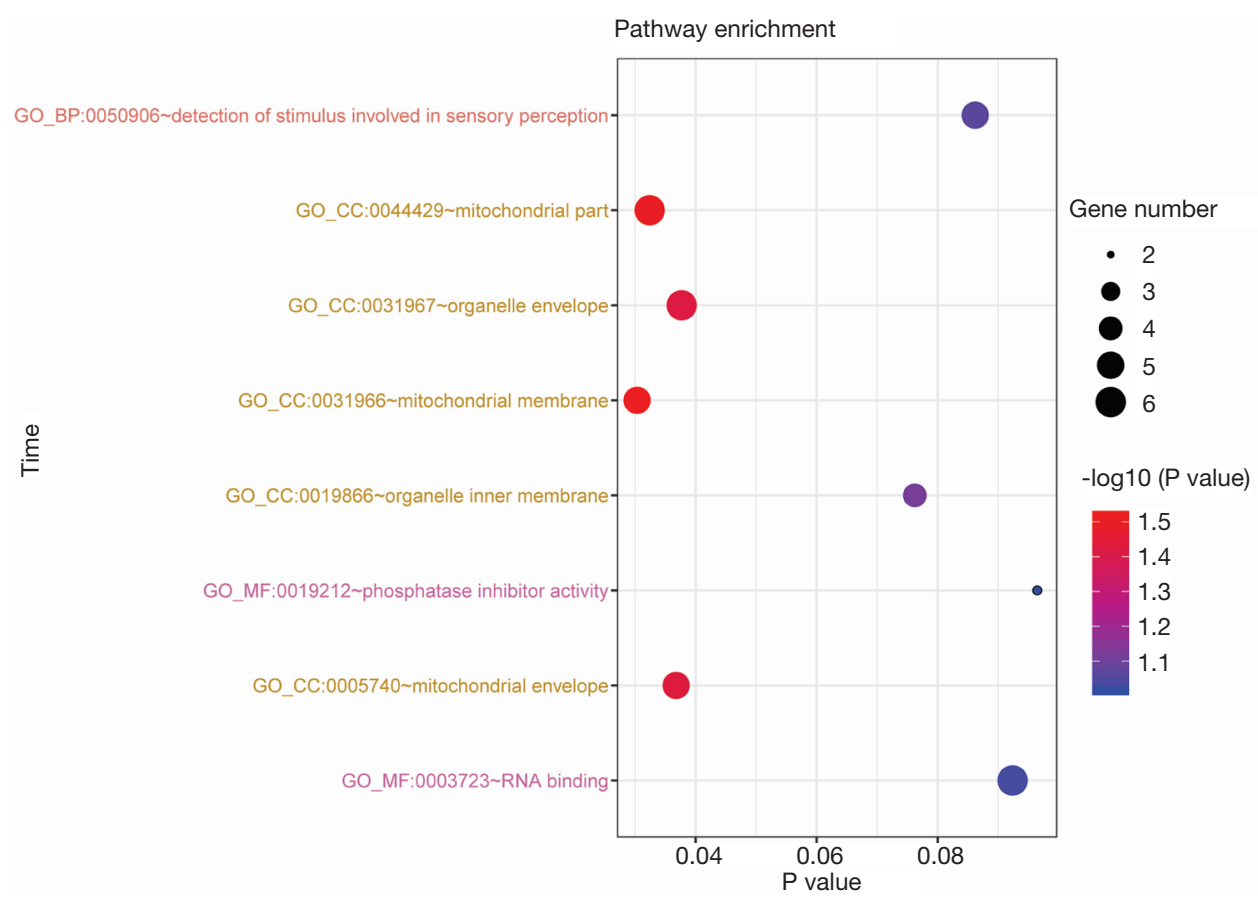

Figure 5 GO and KEGG significant enrichment analysis for DEGs in network. The color of the y-axis indicates the classification of GO terms. Red, biological process (BP); yellow, cellular component (CC); purple, molecular function (MF). 


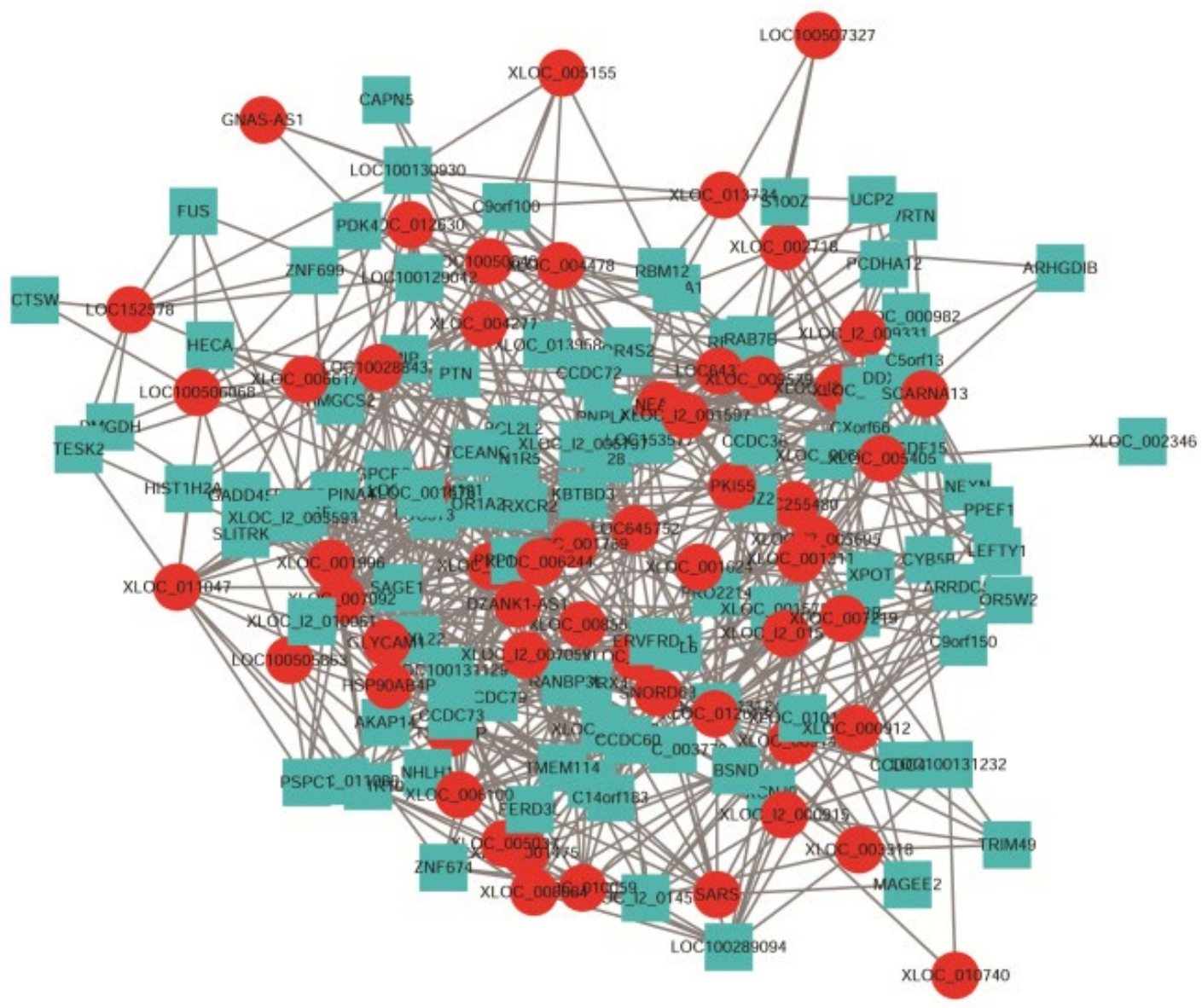

Figure 6 DEGs mRNA-lncRNA network complex and modular analysis. Using the Cytoscape software, a total of 159 DEGs (red circles represent lncRNA, turquoise squares represent mRNA) was filtered into the DEGs network complex.

speckles $(38,39)$. Furthermore, recent studies observed that NEAT1 is upregulated in human CRC tissue and is associated with poor prognosis of CRC, suggesting a critical role in tumor invasion and metastasis. The fact that NEAT1 level is regulated by NF- $\kappa \mathrm{B}$ and STAT3 downstream of the epidermal growth factor receptor (EGFR) signaling pathway makes NEAT1 and its upstream mediators interesting therapeutic targets in malignant tumors (40). Despite potential side effects, blocking NF$\kappa \mathrm{B}$ and STAT3 activity may represent a good approach to suppress tumors overexpressing NEAT1 (41). In chronic inflammatory diseases and cancer, NF- $\kappa \mathrm{B}$ usually appears to be abnormally active and promotes disease and tumor progression by promoting inflammation, preventing differentiation, driving stem cell proliferation and inhibiting apoptosis (42-44). A large body of data supports that NF$\kappa \mathrm{B}$ activity dysregulation plays a critical role in intestinal tumorigenesis, the type of cancer most sensitive to aspirin treatment.

Recent studies have shown that aspirin inhibits the degradation of $\mathrm{I}-\kappa \mathrm{B}$ by blocking the activation of the $\mathrm{NF}-\kappa \mathrm{B}$ pathway to exert antitumor effects in vitro and in animal experiments (45). After being phosphorylated and activated by JAK, STAT3, a member of the STAT family of transcription factors, forms a dimer that is transferred from the cytoplasm to the nucleus, and combines with the promoter of the gene of interest and promotes its expression. In recent years, STAT3 has been shown to promote the occurrence and development of gastrointestinal malignant tumors by regulating the overexpression of Bcl-2, survivin, MMP, VEGF, and other proteins in cell proliferation and anti-apoptosis, tumor invasion and metastasis, and tumor angiogenesis (46). On the other hand, aspirin administration has been demonstrated to reduce the 

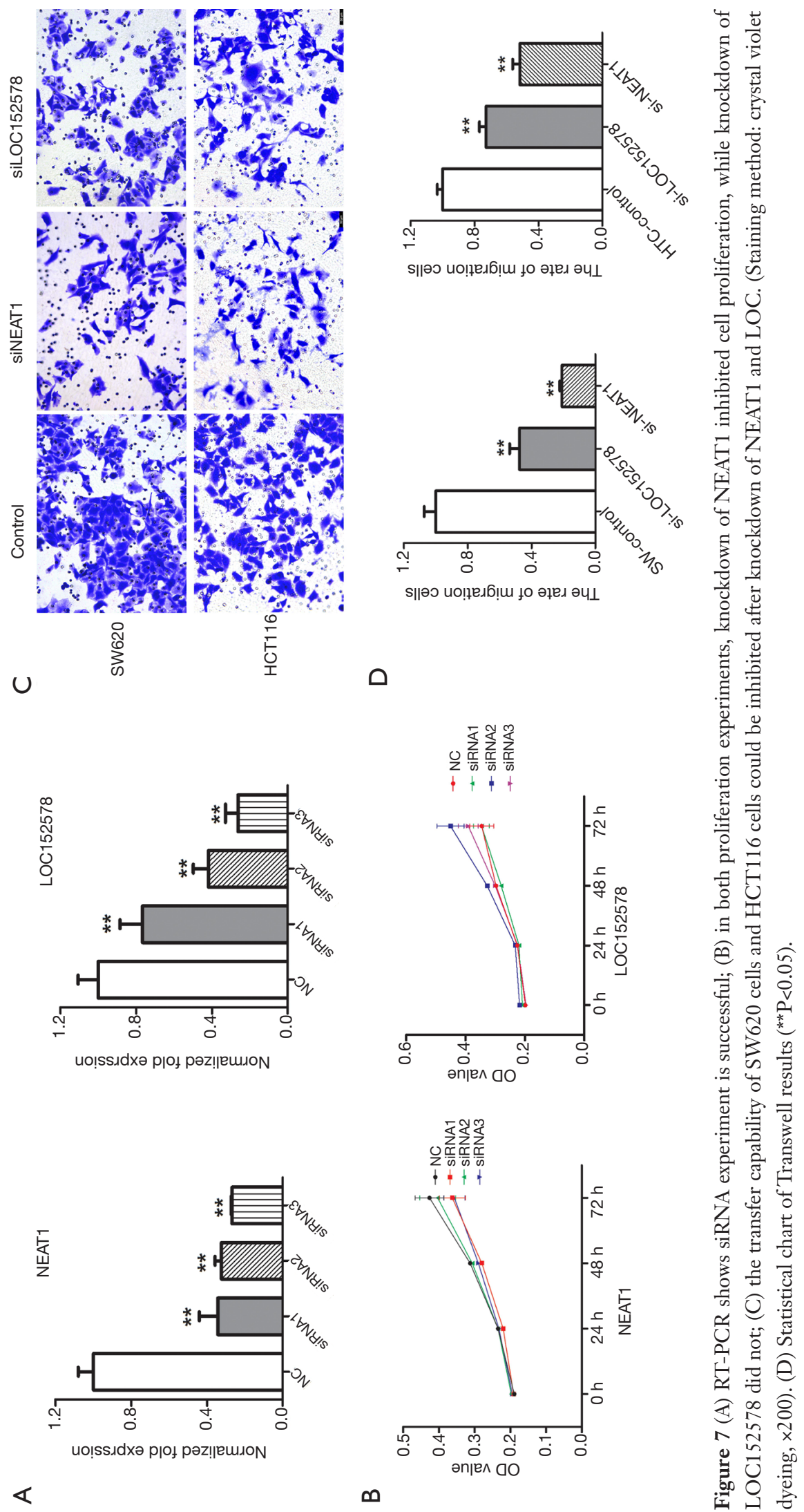

$\varangle$
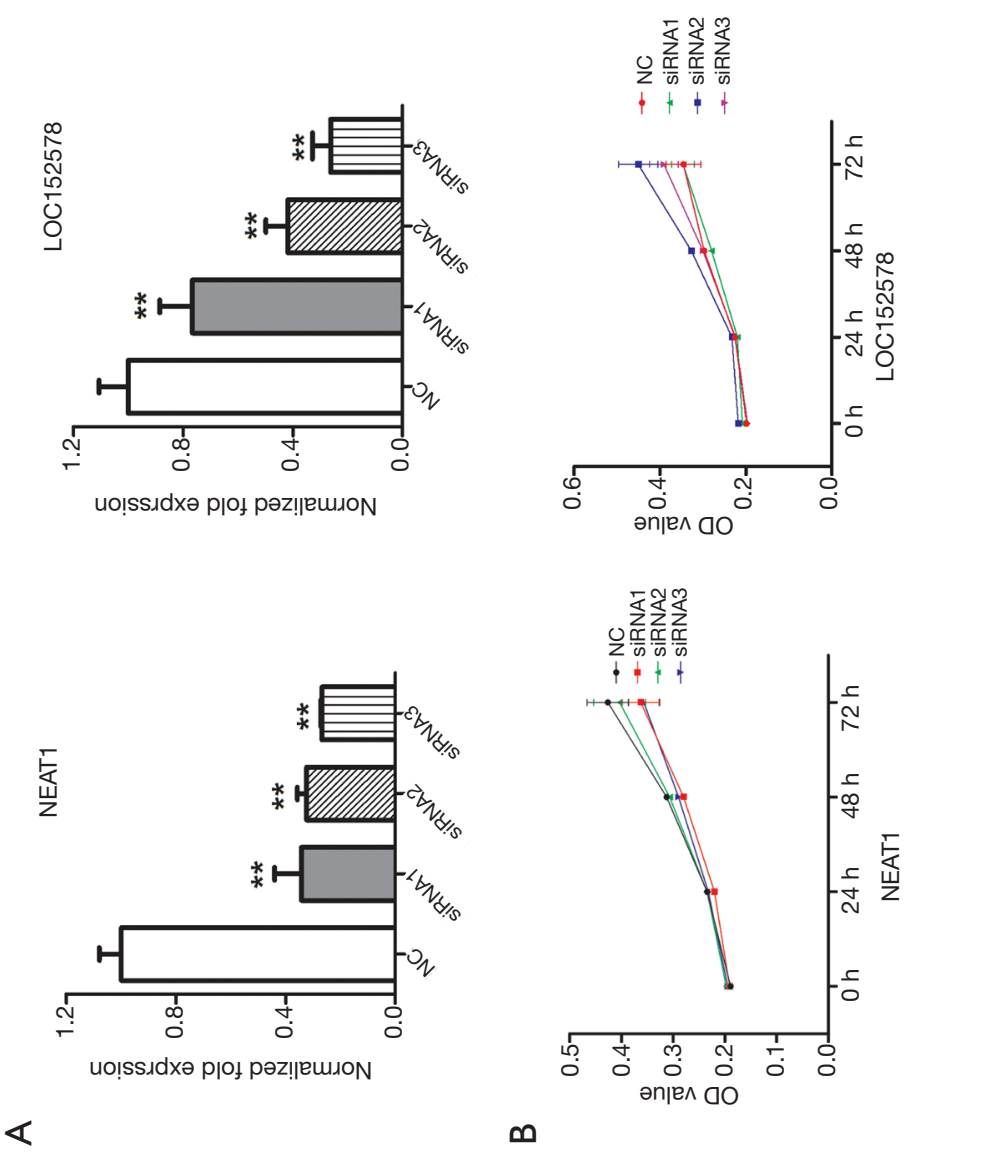

$\infty$

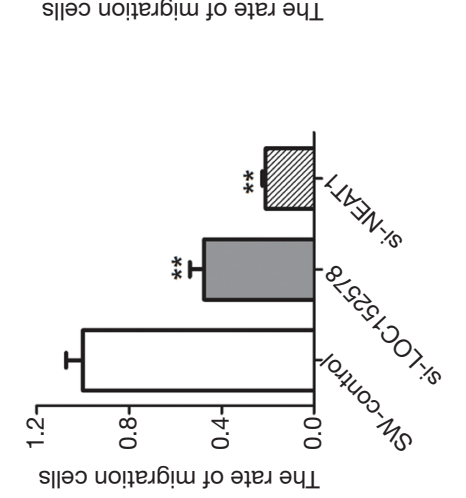

$\square$

î.

(C) Translational Cancer Research. All rights reserved. 
MMP-9 expression and inhibit EMT by blocking STAT3 phosphorylation in other tumors (47). However, the exact mechanism remains nebulous thus far. The findings from the current suggest that NEAT1 is a plausible downstream mediator for aspirin's anticancer effects.

Apart from NEAT1, a novel lncRNA, LOC152578, has also been reported recently for its association with CRC carcinogenesis and postoperative recurrence $(37,48)$. However, its regulatory mechanism has not been reported. We therefore took special interests in this lncRNA and included it in our bioinformatic analyses to evaluate its potential as a biomarker or molecular therapeutic target for CRC. Our study observed downregulation of LOC152578 and upregulation of HMGCS2 in aspirin-treated colon cancer cells.

Mitochondrial 3-hydroxy-3-methylglutaryl-CoA synthase (HMGCS2), a member of the HMG-CoA protein family, is a potential regulatory point in the pathway that converts acetyl-CoA to ketone bodies. However, the role of HMGCS2 in CRC is largely unknown. Recent bioinformatics analyses of TCGA data found that the median survival time of CRC patients with low HMGCS2 expression is significantly shorter than that of those with high HMGCS2 expression (49,50). Zou et al. reported in their most recent paper that HMGCS2 expression was significantly reduced in CRC and was negatively correlated with neovascularization density in CRC (51). Taken together, these findings suggest that HMGCS2, as a tumor suppressor gene, was not only downregulated in CRC but also associated with tumor differentiation. In this study, both LOC152578 and HMGCS2 responded to aspirin treatment in colon cancer cells, with the former being downregulated and the later upregulated, implying a negative correlation between these two RNA molecules. Further studies are needed to confirm if the two responds to aspirin treatment independently or two manifests of a common underlying mechanism.

In this study, we aimed to discover the major role of lncRNA in aspirin's inhibition of colon cancer growth, metastasis, and progression. However, it is worth noting that the mechanism of aspirin in tumor prevention and treatment is still unclear. In order to obtain maximum anticancer benefits, future research needs to focus on accurate lncRNA molecules and reasonable selection and dosage.

\section{Acknowledgments}

Funding: This study was supported by Tibetan Medicine
Regional Collaborative Innovation Center Project (2017XTCX009).

\section{Footnote}

Reporting Checklist: The authors have completed the MDAR checklist. Available at http://dx.doi.org/10.21037/tcr-202248

Conflicts of Interest: All authors have completed the ICMJE uniform disclosure form (available at http://dx.doi. org/10.21037/tcr-20-2248). The authors have no conflicts of interest to declare.

Ethical Statement: The authors are accountable for all aspects of the work in ensuring that questions related to the accuracy or integrity of any part of the work are appropriately investigated and resolved.

Open Access Statement: This is an Open Access article distributed in accordance with the Creative Commons Attribution-NonCommercial-NoDerivs 4.0 International License (CC BY-NC-ND 4.0), which permits the noncommercial replication and distribution of the article with the strict proviso that no changes or edits are made and the original work is properly cited (including links to both the formal publication through the relevant DOI and the license). See: https://creativecommons.org/licenses/by-nc-nd/4.0/.

\section{References}

1. Siegel RL, Miller KD, Jemal A. Cancer Statistics, 2017. CA Cancer J Clin 2017;67:7-30.

2. Jemal A, Thomas A, Taylor M, et al. Cancer statistics, 2002. CA Cancer J Clin 2002;52:23-47.

3. Hardy RG, Meltzer SJ. ABC of colorectal cancer. Molecular basis for risk factors. BMJ 2000;321:886-9.

4. Sancho E, Batlle E, Clevers H. Signaling pathways in intestinal development and cancer. Annu Rev Cell Dev Biol 2004;20:695-723.

5. Carvalho B, Sillars-Hardebol AH, Cindy P. Colorectal adenoma to carcinoma progression is accompanied by changes in gene expression associated with ageing, chromosomal instability, and fatty acid metabolism. Cell Oncol (Dordr) 2012;35:53-63.

6. Long AG, Lundsmith ET, Hamilton KE. Inflammation and Colorectal Cancer. Curr Colorectal Cancer Rep 2017;13:341-51. 
7. Thun MJ, Henley SJ, Patrono C. Nonsteroidal antiinflammatory drugs as anticancer agents: mechanistic, pharmacologic, and clinical issues. J Natl Cancer Inst 2002;94:252-66.

8. Ruder EH, Laiyemo AO, Graubard BI, et al. Nonsteroidal anti-inflammatory drugs and colorectal cancer risk in a large, prospective cohort. Am J Gastroenterol 2011;106:1340-50.

9. Sharpe CR, Collet JP, McNutt M, et al. Nested casecontrol study of the effects of non-steroidal antiinflammatory drugs on breast cancer risk and stage. Br J Cancer 2000;83:112-20.

10. Khuder SA, Mutgi AB. Breast cancer and NSAID use: a meta-analysis. Br J Cancer 2001;84:1188-92.

11. Cuzick J, Otto F, Baron JA, et al. Aspirin and nonsteroidal anti-inflammatory drugs for cancer prevention: an international consensus statement. Lancet Oncol 2009;10:501-7.

12. Bosetti C, Gallus S, La Vecchia C. Aspirin and cancer risk: a summary review to 2007. Recent Results Cancer Res 2009;181:231-51.

13. Flossmann E, Rothwell PM; British Doctors Aspirin Trial and the UK-TIA Aspirin Trial. Effect of aspirin on long-term risk of colorectal cancer: consistent evidence from randomised and observational studies. Lancet 2007;369:1603-13.

14. Rothwell PM, Wilson M, Price JP, et al. Effect of daily aspirin on risk of cancer metastasis: a study of incident cancers during randomised controlled trials. Lancet 2012;379:1591-601.

15. Khan MN, Lee YS. Cyclooxygenase inhibitors: scope of their use and development in cancer chemotherapy. Med Res Rev 2011;31:161-201.

16. Huang WW, Hsieh KP, Huang RY, et al. Role of cyclooxygenase-2 inhibitors in the survival outcome of colorectal cancer patients: A population-based cohort study. Kaohsiung J Med Sci 2017;33:308-14.

17. Wang KC, Chang HY. Molecular mechanisms of long noncoding RNAs. Mol Cell 2011;43:904-14.

18. Langley RE. Clinical evidence for the use of aspirin in the treatment of cancer. Ecancermedicalscience 2013;7:297.

19. Rutenberg-Schoenberg M, Sexton AN, Simon MD. The Properties of Long Noncoding RNAs That Regulate Chromatin. Annu Rev Genomics Hum Genet 2016;17:69-94.

20. Yu SY, Tang L, Zhou SH. Long Noncoding RNAs: New Players in Ischaemia-Reperfusion Injury. Heart Lung Circ 2018;27:322-32.
21. Hung T, Wang Y, Lin MF, et al. Extensive and coordinated transcription of noncoding RNAs within cell-cycle promoters. Nat Genet 2011;43:621-9.

22. Iyer MK, Niknafs YS, Malik R, et al. The landscape of long noncoding RNAs in the human transcriptome. Nat Genet 2015;47:199-208.

23. Quan J, Pan X, Zhao L, et al. LncRNA as a diagnostic and prognostic biomarker in bladder cancer: a systematic review and meta-analysis. Onco Targets Ther 2018;11:6415-24.

24. Tian T, Wang M, Lin S, et al. The Impact of lncRNA Dysregulation on Clinicopathology and Survival of Breast Cancer: A Systematic Review and Meta-analysis. Mol Ther Nucleic Acids 2018;12:359-69.

25. Bhan A, Soleimani M, Mandal SS. Long Noncoding RNA and Cancer: A New Paradigm. Cancer Res 2017;77:3965-81.

26. Kawasaki Y, Miyamoto M, Oda T, et al. The novel lncRNA CALIC upregulates AXL to promote colon cancer metastasis. EMBO Rep 2019;20:e47052.

27. Peng CL, Zhao XJ, Wei CC, et al. LncRNA HOTAIR promotes colon cancer development by downregulating miRNA-34a. Eur Rev Med Pharmacol Sci 2019;23:5752-61.

28. Ding D, Li C, Zhao T, et al. LncRNA H19/miR-29b-3p/ PGRN Axis Promoted Epithelial-Mesenchymal Transition of Colorectal Cancer Cells by Acting on Wnt Signaling. Mol Cells 2018;41:423-35.

29. Zhu Y, Yang C, Weng M, et al. Identification of TMEM208 and PQLC2 as reference genes for normalizing mRNA expression in colorectal cancer treated with aspirin. Oncotarget 2017;8:22759-71.

30. Chan AT, Giovannucci EL, Meyerhardt JA, et al. Long-term use of aspirin and nonsteroidal antiinflammatory drugs and risk of colorectal cancer. JAMA 2005;294:914-23.

31. Rothwell PM, Wilson M, Elwin CE, et al. Long-term effect of aspirin on colorectal cancer incidence and mortality: 20-year follow-up of five randomised trials. Lancet 2010;376:1741-50.

32. Alfonso L, Ai G, Spitale RC, et al. Molecular targets of aspirin and cancer prevention. Br J Cancer 2014;111:61-7.

33. Hua H, Zhang H, Kong Q, et al. Complex roles of the old drug aspirin in cancer chemoprevention and therapy. Med Res Rev 2019;39:114-45.

34. Liu J, Wan L, Lu K, et al. The Long Noncoding RNA MEG3 Contributes to Cisplatin Resistance of Human Lung Adenocarcinoma. PLoS One 2015;10:e0114586. 
35. Malek E, Jagannathan S, Driscoll JJ. Correlation of long non-coding RNA expression with metastasis, drug resistance and clinical outcome in cancer. Oncotarget 2014;5:8027-38.

36. Schmitt AM, Chang HY. Long Noncoding RNAs in Cancer Pathways. Cancer Cell 2016;29:452-63.

37. Shi J, Li X, Zhang F, et al. Circulating lncRNAs associated with occurrence of colorectal cancer progression. Am J Cancer Res 2015;5:2258-65.

38. Clemson CM, Hutchinson JN, Sara SA, et al. An architectural role for a nuclear noncoding RNA: NEAT1 RNA is essential for the structure of paraspeckles. Mol Cell 2009;33:717-26.

39. Anantharaman A, Jadaliha M, Tripathi V, et al. Paraspeckles modulate the intranuclear distribution of paraspeckle-associated Ctn RNA. Sci Rep 2016;6:34043.

40. Chen Q, Cai J, Wang Q, et al. Long Noncoding RNA NEAT1, Regulated by the EGFR Pathway, Contributes to Glioblastoma Progression Through the WNT/ $\beta$-Catenin Pathway by Scaffolding EZH2. Clin Cancer Res 2018;24:684-95.

41. Grivennikov SI, Karin M. Dangerous liaisons: STAT3 and NF-kappaB collaboration and crosstalk in cancer. Cytokine Growth Factor Rev 2010;21:11-9.

42. Perkins ND. NF-kappaB: tumor promoter or suppressor? Trends Cell Biol 2004;14:64-9.

43. DiDonato JA, Mercurio F, Karin M. NF-kappaB and the link between inflammation and cancer. Immunol Rev, 2012;246:379-400.

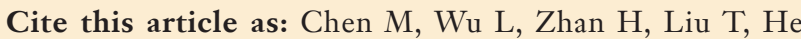
Y. Aspirin induced long non coding RNA suppresses colon cancer growth. Transl Cancer Res 2021;10(5):2055-2069. doi: $10.21037 /$ tcr-20-2248
44. Vlahopoulos SA, Cen O, Hengen N, et al. Dynamic aberrant NF-kappaB spurs tumorigenesis: a new model encompassing the microenvironment. Cytokine Growth Factor Rev 2015;26:389-403.

45. Liao D, Zhong L, Duan T, et al. Aspirin Suppresses the Growth and Metastasis of Osteosarcoma through the NFkappaB Pathway. Clin Cancer Res 2015;21:5349-59.

46. Shi M, Liu D, Duan H, et al. Catecholamine up-regulates MMP-7 expression by activating AP-1 and STAT3 in gastric cancer. Mol Cancer 2010;9:269.

47. Han Y, Guo W, Ren T, et al. Tumor-associated macrophages promote lung metastasis and induce epithelial-mesenchymal transition in osteosarcoma by activating the COX-2/STAT3 axis. Cancer Lett 2019;440441:116-25.

48. Yamada A, Yu P, Lin W, et al. A RNA-Sequencing approach for the identification of novel long non-coding RNA biomarkers in colorectal cancer. Sci Rep 2018;8:575.

49. Camarero N, Mascaró C, Mayordomo C, et al. Ketogenic HMGCS2 Is a c-Myc target gene expressed in differentiated cells of human colonic epithelium and downregulated in colon cancer. Mol Cancer Res 2006;4:645-53.

50. Kim JT, Li C, Weiss HL, et al. Regulation of Ketogenic Enzyme HMGCS2 by Wnt/ $\beta$-catenin/PPAR $\gamma$ Pathway in Intestinal Cells. Cells 2019;8:1106.

51. Zou K, Hu Y, Li M, et al. Potential Role of HMGCS2 in Tumor Angiogenesis in Colorectal Cancer and Its Potential Use as a Diagnostic Marker. Can J Gastroenterol Hepatol 2019;2019:8348967. 